\title{
Prioritized Maps of Space in Human Frontoparietal Cortex
}

\author{
Trenton A. Jerde, ${ }^{1}$ Elisha P. Merriam, ${ }^{2}$ Adam C. Riggall, ${ }^{1}$ James H. Hedges, ${ }^{2}$ and Clayton E. Curtis ${ }^{1,2}$ \\ ${ }^{1}$ Department of Psychology and ${ }^{2}$ Center for Neural Science, New York University, New York, New York 10003
}

Priority maps are theorized to be composed of large populations of neurons organized topographically into a map of gaze-centered space whose activity spatially tags salient and behaviorally relevant information. Here, we identified four priority map candidates along human posterior intraparietal sulcus (IPS0-IPS3) and two along the precentral sulcus (PCS) that contained reliable retinotopically organized maps of contralateral visual space. Persistent activity increased from posterior-to-anterior IPS areas and from inferior-to-superior PCS areas during the maintenance of a working memory representation, the maintenance of covert attention, and the maintenance of a saccade plan. Moreover, decoders trained to predict the locations on one task (e.g., working memory) cross-predicted the locations on other tasks (e.g., attention) in superior PCS and IPS2, suggesting that these patterns of maintenance activity may be interchangeable across the tasks. Such properties make these two areas in frontal and parietal cortex viable priority map candidates.

\section{Introduction}

Recently, several lines of evidence from a variety of disciplines have converged on a theory positing that activity in the frontal and parietal cortices constitutes maps of prioritized space (Itti and Koch, 2001; Thompson and Bichot, 2005; Fecteau and $\mathrm{Mu}-$ noz, 2006; Serences and Yantis, 2006; Bisley and Goldberg, 2010). In this conceptual framework, the activity of neurons in priority maps rank important locations in the visual field according to their attentional priority (Bisley and Goldberg, 2010). Priority maps are dynamically sculpted by the saliency, or conspicuousness, of bottom-up, feedforward information from early visual neurons about the physical features of stimuli (Itti and Koch, 2001), combined with goal-relevant, top-down information from higher association cortices (Serences and Yantis, 2007). Activity in a priority map could be used to select between competing representations of actions in the motor system or between competing representations of objects in the visual system.

Presumably, the populations of neurons comprising maps of prioritized space are organized in a retinal topographic manner, with each neuron coding for different but overlapping portions of the visual field (Itti and Koch, 2001). Recently, topographic maps of contralateral space have been reported in humans beyond visual cortex in posterior parietal cortex (PPC) and prefrontal cortex (PFC) (Sereno et al., 2001; Schluppeck et al., 2005; Silver et al., 2005; Hagler and Sereno, 2006; Kastner et al., 2007; Swisher et al., 2007). Here we ask the following: might these

Received Aug. 9, 2012; revised Sept. 7, 2012; accepted 0ct. 2, 2012.

Author contributions: T.A.J. and C.E.C. designed research; T.A.J., A.C.R., and C.E.C. performed research; T.A.J., E.P.M., A.C.R., J.H.H., and C.E.C. analyzed data; T.A.J. and C.E.C. wrote the paper.

This work was supported by National Institutes of Health Grants EY016407 (C.E.C.) and EY019221 (T.A.J.). We thank the Center for Brain Imaging at New York University and their staff for support during data collection, David Heeger and Justin Gardner for software used for stimulus presentation and data analysis, and Bonnie Lawrence for comments on this manuscript.

The authors declare no competing financial interests.

Correspondence should be addressed to Clayton E. Curtis, Department of Psychology, New York University, 6 Washington Place, New York, NY 10003. E-mail: clayton.curtis@nyu.edu.

DOI:10.1523/JNEUROSCI.3810-12.2012

Copyright $\odot 2012$ the authors $\quad 0270-6474 / 12 / 3217382-09 \$ 15.00 / 0$ topographic areas contain populations of neurons whose activity prioritizes space? To answer this question, we first identified candidate priority maps by defining topographically organized areas of PPC and PFC. To identify topographic maps, we used functional magnetic resonance imaging (fMRI) to measure traveling waves of blood oxygenation level-dependent (BOLD) activity evoked by the systematic shifting of the subject's focus of covert attention around the visual field.

Then, we tested whether the pattern of activity in candidate priority maps is indistinguishable across different spatial cognitive tasks. Theoretically, priority maps may be agnostic with regard to what caused the priority. To test this possibility, we first compared BOLD signal changes in candidate priority maps while subjects maintained a location in working memory, maintained their attention covertly in the periphery, and maintained a saccade plan. Second, we tested whether we could decode the location of memory, attention, and intention based on the multivoxel pattern of delay-period activity. Third, we tested whether the classifiers generalize across spatial tasks. We trained classifiers on one task (e.g., working memory) and tested its predictive validity on the other tasks (e.g., attention and intention). We found persistent activity in some, but not all, topographic areas in frontal and parietal cortex during working memory, attention, and motor planning. Moreover, decoders trained to predict the locations on one task cross-predicted the locations on other tasks in superior precentral sulcus (sPCS) and intraparietal sulcus 2 (IPS2), suggesting that these patterns of maintenance activity may be interchangeable across the tasks. We conclude that these two topographic areas may form prioritized maps of space.

\section{Materials and Methods}

Subjects. Four psychophysically experienced, neurologically healthy, right-handed (mean laterality index, 85; range, 75-100) (Oldfield, 1971) subjects (three men, one woman) who were naive to the hypotheses, 25-40 years old, with normal or corrected-to-normal vision, participated. Subjects gave written informed consent, and the Institutional Review Board at New York University approved all procedures.

Oculomotor methods. Eye position was monitored in the scanner using an eye tracker (SR Research). Eye position data were preprocessed and 
a

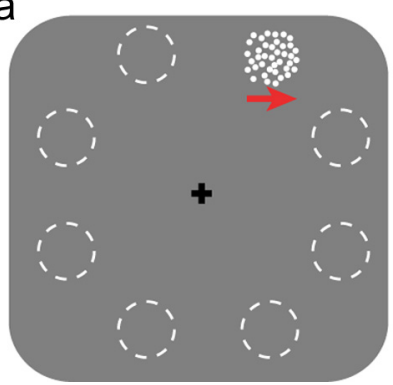

$\mathrm{b}$

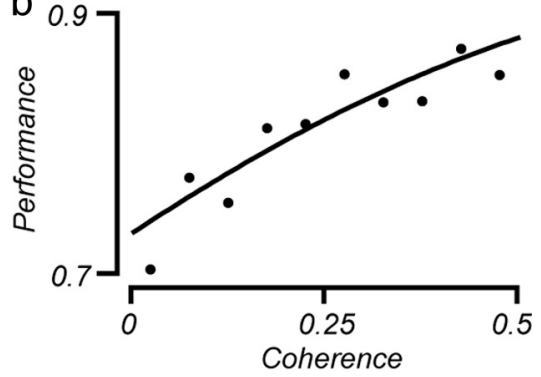

Figure 1. $\quad \boldsymbol{a}$, Task. Dots moving at various levels of coherence to the left or right were visible in one of eight possible apertures (dashed circles were invisible to subject) that were covertly attended. The subject indicated with a button press the direction of coherent motion (e.g., right, red arrow was invisible to subject). The position of the aperture of dot motion moved sequentially in a clockwise or counterclockwise direction. $\boldsymbol{b}$, A staircase that adjusted the fraction of dots moving coherently controlled task difficulty, and performance predictably improved with coherence.

scored offline with custom software. For the dot motion discrimination task, we analyzed eye movements in two ways. First, we assessed whether the eyes systematically deviated toward the direction of the aperture containing the dots by calculating correlations between eye position and the locations of the apertures. This correlation tested whether the eyes, although fixating, deviated systematically toward the location of the dot motion apertures. Second, we calculated the number of saccades made in the direction of the dot motion aperture. Saccades were defined as eye movements with amplitude of $\geq 1^{\circ}$ and a minimum velocity of $30 \%$ s. For the event-related studies, we measured eye position to confirm that fixation was maintained during the delay periods of each task.

Functional imaging. MRI data were collected using a $3 \mathrm{~T}$ head-only scanner (Allegra; Siemens) at the Center for Brain Imaging at New York University. Images were acquired using a custom four-channel phased array receive coil (NOVA Medical) placed over lateral frontal and parietal cortices. In the topographic mapping experiment, a series of volumes was acquired using a $2^{\star}{ }^{\star}$-sensitive echo planar imaging (EPI) pulse sequence [repetition time (TR), $2000 \mathrm{~ms}$; echo time, $30 \mathrm{~ms}$; flip angle, $75^{\circ}$; 36 slices; $3 \times 3 \times 3 \mathrm{~mm}$ voxels]. In the event-related experiments, we used a shorter TR of $1500 \mathrm{~ms}$, which resulted in fewer slices (24) and smaller coverage that targeted the dorsal frontal and parietal cortex (early visual cortex was not covered). T1-weighted low-resolution anatomical images were collected at the beginning of each scanning session using the same slice prescriptions as the functional data. High-resolution $(1 \times 1 \times$ $1 \mathrm{~mm}$ ) T1-weighted scans were acquired for registration, segmentation, and display.

Topographic mapping. To identify topographic maps and assess their reliability, subjects participated in two topographic mapping sessions. The visual stimuli and task used standard traveling wave methods (Engel et al., 1994) to identify topographic areas. Stimuli were generated using MATLAB (MathWorks) and MGL (available at http://justingardner. net $/ \mathrm{mgl}$ ) on a Macintosh computer and displayed on a screen in the magnet bore. We evoked traveling waves of activity in topographic areas by shifting covert attention around the visual field. Specifically, the apertures containing moving dots were presented one at a time in eight equally spaced locations around the periphery of central fixation (Fig. 1a). Each aperture consisted of 120 small white dots moving $\left(3.125^{\circ} / \mathrm{s}\right)$ independently within an invisible circular aperture $\left(6.25^{\circ}\right.$ diameter $)$ centered $8^{\circ}$ from the fixation cross for $1750 \mathrm{~ms}$ before moving to the next position (e.g., clockwise). Subjects indicated, with a button press, the direction (left or right) of coherently moving dots. Task difficulty was controlled by a psychophysical staircase (one-up, two-down Levitt staircase; accuracy of $\sim 75 \%$ ) (Wetherill and Levitt, 1965) that changed the coherence of moving dots and ensured that covert attention was focused and sustained toward each aperture of moving dots. The staircase equated performance across runs and scan sessions for each subject and across subjects as a whole. Each 320 s run consisted of 20 cycles, in which one cycle consisted of eight apertures presented around the periphery, at 16 s per cycle, critically pushing the task frequency beyond the bulk of the $1 / \mathrm{f}$ structured noise of the MRI. Subjects performed 6-14 runs per scanning session.

The average time-series data were analyzed by fitting a sinusoid at the task frequency to the time series at each voxel and computing the coherence and the phase of the best-fitting sinusoid. The coherence value quantified signal to noise (Engel et al., 1997), taking a value near 1 when the fMRI signal modulation at the period of the stimulus cycle was large relative to the noise and a value near 0 when the signal modulation was small compared with the noise. The response phase measures the temporal delay of the fMRI signal relative to the beginning of the dot motion stimulus cycle and therefore corresponded to the polar-angle component of the topographic map. Maps of coherence and phase were displayed on flattened representations of the cortical surface.

The motion discrimination task revealed at least four topographically organized areas within PPC and two areas within PFC. Although the visual stimulation in the motion discrimination task subtended a much smaller portion of the visual field than that typically used in retinotopic mapping procedures, visual cortex V1, V2, and V3 maps were clearly identifiable in occipital cortex (Fig. 2). Boundaries between topographic areas were drawn on flat maps, guided by the spatial organization of the response phases and vertical meridians, the locations of the areas relative to each other, and the coherence values. Circular correlation was used to quantify the similarity of phase values within the overlapping voxels in topographic areas between the two sessions (Jammalamadaka and Sengupta, 2001). To assess the significance of circular correlations for topographic areas, the phase values from the two sessions were shuffled (i.e., randomly reassigned to different voxels) to simulate the null hypothesis that the two maps were unrelated, and correlations were recomputed. This process was repeated 10,000 times to obtain a null distribution of correlation values. $p$ values were determined by comparison with the null distribution as $P_{\text {boot }}=\left(1+\#\left\{r_{\text {null }} \geq r\right\}\right) /(n+1)$, where $r_{\text {null }}$ is the correlation from randomized voxels and $n$ is the number of iterations.

Event-related $f M R I$ studies of spatial cognition. The same subjects participated in three event-related fMRI experiments that involved covert attention, working memory, and motor planning. Key details about these tasks are given below. The trial structures (e.g., timing, variable delay lengths, stimuli, feedback, etc.) were virtually identical across the different tasks. Here, we focus on activity during the delay periods of these tasks, in which spatial priorities driven by working memory, attention, or saccade planning were maintained across long and variable delay periods, before any overt motor response. In the spatial attention task, subjects fixated a central point that was flanked by a circle and a square (both $1^{\circ}$ of visual angle) on a black background. These stimuli were $\pm 5.5^{\circ}$ from the center of the screen on the horizontal meridian. The fixation point changed to white for $1500 \mathrm{~ms}$ to signal the beginning of a new trial and was then replaced to match one of the two flanking shapes, which instructed subjects to shift attention to the matching shape. Subjects maintained attention at the cued location for a long, variable, and unpredictable duration (7.5-13.5 s). During this interval, the attended target occasionally and unpredictably dimmed slightly in luminance, resulting in a contrast change that was near threshold for $75 \%$ performance accuracy. To ensure active maintenance of attention at the cued location throughout the interval, subjects counted the number of times that the target dimmed. The attended target dimmed between one and four times for $100 \mathrm{~ms}$ during the delay. After the delay, the fixation point turned to green $(3000 \mathrm{~ms})$, instructing subjects to report the number of target dims by pressing a corresponding button on a button box. In the spatial working memory task, a trial began with a preparation cue (white dot, $1500 \mathrm{~ms}$ ) at the center of the screen, indicating the beginning of a new trial. Then a sample stimulus (cyan square, $150 \mathrm{~ms}$ ) was flashed from 5 to $15^{\circ}$ to the left or right and from 4 to $5^{\circ}$ above or below the central fixation point. Subjects remembered the location of the sample for the duration of a long, variable, and unpredictable delay period (7.5-13.5 s). After the delay, a test stimulus appeared at or near the previous location 


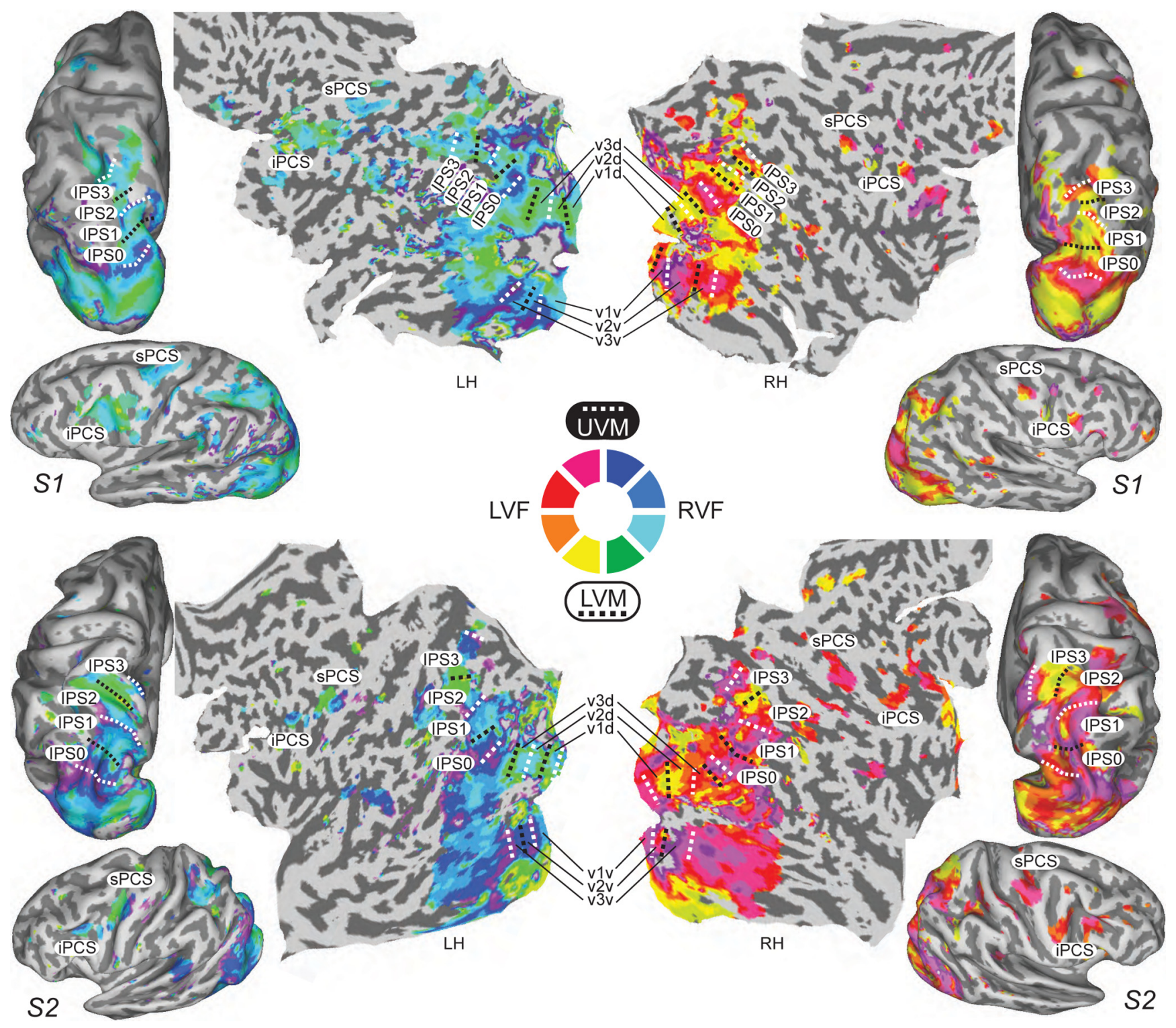

Figure 2. Topographic maps from two subjects. On both inflated and flattened cortices, phase values are represented. As indicated in the central color wheel key, cool colors reflect the right visual field (RVF), and warm colors reflect the left visual field (LVF). The borders of topographic areas in occipital and parietal cortex are demarked by dotted black lines reflecting the lower visual meridian (LVM) and dotted white lines reflecting the upper visual meridian (UVM). In both subjects, anterior and dorsal to early visual areas V1-V3, four topographic areas are found along the caudal-rostral intraparietal sulcus (IPSO -IPS3). In the PFC, two topographic areas are found along the dorsal—ventral PCS (sPCS and PPCS). LH, Left hemisphere; RH, right hemisphere.

of the sample, and subjects indicated, with a button press using their right index or ring finger, whether it matched the location of the sample. A staircase adjusted the distance between the locations of the samples and non-matching test stimuli to keep performance near $75 \%$ accuracy. In the motor intention task, a trial began with a preparation cue (white dot, $1500 \mathrm{~ms}$ ) at the center of the screen indicating the beginning of a new trial. A target stimulus ( $1^{\circ}$ cyan square) appeared from 5 to $15^{\circ}$ to the left or right and from 4 to $5^{\circ}$ above or below the fixation point. Subjects maintained fixation during a long, variable, and unpredictable delay period $(7.5-13.5 \mathrm{~s})$, during which they were instructed to plan the eye movement and remain in a ready state to execute it when the fixation point disappeared. Each imaging session consisted of four runs, yielding 64 trials per condition, per subject.

The EPI volumes were motion corrected, slice-timing adjusted, and highpass filtered at $0.01 \mathrm{~Hz}$. Time courses of BOLD signal change were extracted from the topographic maps defined in the topographic mapping experiments for each subject, time locked to trial onset, and averaged across trials. Importantly, these time courses only included the TRs up to the end of the delay, so as not to contaminate the estimation with activity evoked by the motor response after the delay. A repeated-measures ANOVA, with topographic area and cognitive task as factors, was used to test for significant delay period activity and differences in delay period activity. For the laterality index, delay-period activity was defined as the average of the time points from $6 \mathrm{~s}$ after the sample until the end of the variable delay period. Contralateral activation was defined as activation in the left hemisphere maps when the right visual field was prioritized (i.e., the memoranda, locus of attention, or the goal of the planned saccade was to the right), plus activation in the right maps when the left visual field was prioritized. Ipsilateral activation was defined as the opposite. The laterality estimates were plotted against each other with contralateral values on the $y$-axis and ipsilateral values on the $x$-axis. Furthermore, we calculated a contralaterality index (CI) for each topographic map as the contrast ratio between contralateral and ipsilateral activity $[($ contralateral - ipsilateral $) /($ contralateral + ipsilateral $)]$. Onetailed $t$ tests were used to test for CIs greater than zero in each topographic area for each cognitive task.

We used multivoxel pattern analysis to test whether delay period specific activity in topographic PPC and PFC regions contained information about the location of prioritized space during the three tasks. Specifically, 
a

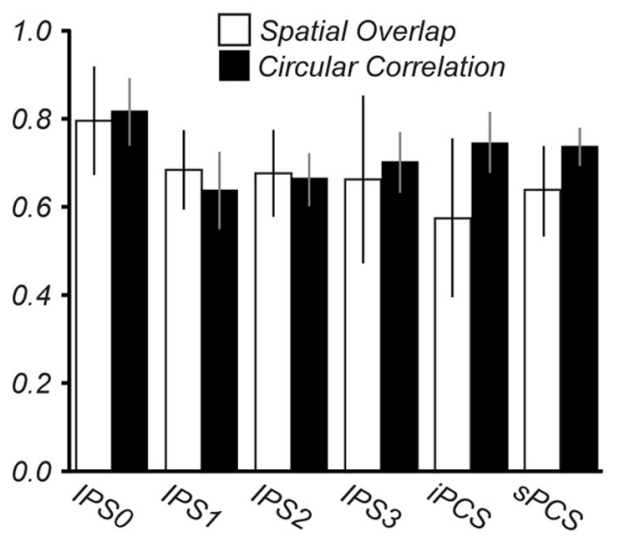

b

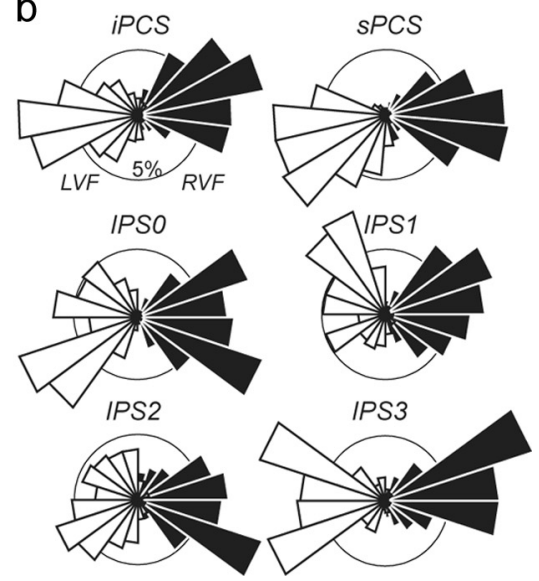

Figure 3. $\boldsymbol{a}$, Reliability of topographic areas. White bars (spatial overlap) reflect the mean \pm SD number of voxels identified in each topographic area across both sessions. Black bars reflect the mean \pm SD circular correlation of phase values in each topographic area across both sessions. $\boldsymbol{b}$, Radial histograms showing the distribution of preferred visual field angles as a percentage of all voxels in each topographic area across the four subjects. White bars are from the right hemisphere, and black bars are from the left hemisphere. LVF, left visual field; LVM, lower visual meridian.

multivoxel patterns of BOLD activity in topographic areas IPS0-IPS3 and sPCS/inferior PCS (iPCS) were assessed in a within-task decoding analysis (e.g., a classifier trained on covert attention data predicts the direction of covert attention) and an across-task decoding analysis (e.g., a classifier trained on covert attention data predicts the location of spatial working memory). The epochs used for analysis were shifted by $4 \mathrm{~s}$ to adjust for the hemodynamic lag, and the BOLD signal was averaged throughout the delay period to yield a single delay period estimate for each trial for each voxel in the topographic maps. Logistic regression, commonly used in the machine-learning field and well suited for highdimensional fMRI data (Pereira et al., 2009), was used to classify whether the left or right visual field was prioritized. We used a conservative penalty $(\lambda=50)$ to reduce the contribution from less informative voxels. For within-task decoding, classification accuracy was determined using leave-two-trials-out cross-validation, in which the classifier was repeatedly trained on data from all but two trials and then tested on the left-out trials. For across-task decoding, the classifier was trained on all of the data from one task and tested on each trial of the other two tasks. Significance was determined by permutation tests that shuffled the condition labels and reran the classification procedures 1000 times to create null distributions. Then, classifier accuracies were compared with thresholds that correspond to $p=0.05$ (two-sided) in the null accuracy distributions. To be considered significant, the group mean accuracy and three of four subjects had to have accuracies above threshold.

\section{Results}

\section{Topographic mapping}

Psychophysics and oculomotor behavior

The motion discrimination task was designed to be demanding and required sequential shifts of covert attention (Fig. 1a). Subjects performed at $82 \pm 3 \%$ (mean $\pm \mathrm{SD}$ ) accuracy in correctly detecting the direction of coherently moving dots, and, as expected, performance improved as the staircase increased the fraction of the dot motion coherence (Fig. 1b). Importantly, this manipulation allowed us to maintain consistent levels of difficulty across time and across individuals.

The subjects were experienced in psychophysical and fMRI tasks. Eye tracking confirmed that the topographic mapping could not have been driven by gaze shifts. Gaze position remained at central fixation, and the cross-correlation between drifts from the fixation cross (almost always $<1^{\circ}$ ) and the position of the dot motion aperture was small (mean \pm SE $r=0.09 \pm$
0.05). Moreover, subjects rarely ( $4.4 \%$ of all dot motion epochs) made saccades $>1^{\circ}$ toward the dot motion apertures.

Topographic maps in PPC and PFC Our first goal was to identify candidate priority maps by defining topographic areas of PFC and PPC. To map the representations of the visual field, we projected the phase values of coherence-thresholded voxels onto the cortical surface of the PPC and PFC for each subject. The color wheel in Figure 2 shows the phase of the fMRI response that corresponds to a preferred angular position in the visual field. Overall, responses were highly lateralized such that the left visual field (warm colors) was represented in the right hemisphere, and the right visual field (cool colors) was represented in the left hemisphere. Responses to the lower and upper vertical meridians were represented in both hemispheres. Phase reversals in orientation were used to demarcate the boundaries of individual topographic maps, as they are in visual cortex (Sereno et al., 1995). This mapping procedure yielded a number of contiguous topographic areas. In PPC, four areas (IPS0-IPS3) were readily identifiable in both hemispheres of all subjects along the IPS. Each of these areas consisted of a representation of the entire contralateral visual field. Figure 2 shows the topographic maps for two representative subjects 1 and 2. Additional topographic areas in PPC were observed for a subset of subjects or sometimes in only one hemisphere. These areas are likely those identified in other studies as IPS4-IPS5 (Swisher et al., 2007; Konen and Kastner, 2008). We focus on IPS0-IPS3 because these maps were the most robust and were found in all subjects and hemispheres.

In PFC, two topographic maps were identified in both hemispheres of all subjects. Figure 2 shows the topography in PFC for two subjects on inflated cortical surface reconstructions. The superior map was located at the intersection of the sPCS and the caudal portion of the superior frontal sulcus. A second topographic map was observed along the caudal convexity of the middle frontal gyrus delving into the iPCS and inferior frontal sulcus. Both of these maps are consistent with previously identified topographic maps in PFC (Hagler and Sereno, 2006; Kastner et al., 2007).

\section{Map reliability across sessions}

Each subject was scanned in two sessions to assess the reliability of the topographic maps. First, two of the authors independently defined the topographic areas each using data from only one of the sessions. The percentage of overlapping voxels was calculated in relation to the total number of voxels. This method measures the extent to which the maps in two sessions covered the same cortical tissue. The majority of the same voxels $(58-80 \%)$ were identified in both sessions (Fig. 3a). The voxels toward the edges of topographic areas tended to be the most likely to be included in only one session, especially for PFC areas in which boundaries are determined by coherence thresholds, not phase reversals associated with adjacent areas. Second, circular correlation was used to quantify the extent to which voxel phase values in the maps were the same across the two sessions (Fig. 3a) (Jammalamadaka and Sengupta, 2001). The correlations in all topographic regions ranged from 0.62 to 0.81 and were highly significant $(p<$ 
0.0001). Overall, the topographic maps in PPC and PFC were reliably defined and stable over time.

Distribution of preferred visual field angles We visualized the distribution of visual field preferences of each topographic area by plotting the phases of all voxels in radial histograms (Fig. 3b). For IPS0-IPS3, the phases were concentrated around the contralateral horizontal meridian and underrepresented near the vertical meridians. This pattern of compression in IPS is similar to that reported previously (Silver et al., 2005; Swisher et al., 2007) and extends beyond IPS into other occipital visual areas (Press et al., 2001; Larsson and Heeger, 2006; Winawer et al., 2010) and even in the lateral geniculate nucleus of the thalamus (Schneider et al., 2004). Such an anisotropic distribution, with a lesser representation at the vertical meridians, may result from a variety of sources. By using the maximum phase value of each voxel, we are mapping the weighted center, and not the extent, of the population response field. Pooling attributable to partial volume effects of fMRI voxel size and the inevitable spatial blurring introduced by MR sampling and hemodynamics (Engel et al., 1997) will skew the estimated phase away from the vertical meridian boundary (Larsson and Heeger, 2006). Consistent with previous findings (Hagler and Sereno, 2006; Kastner et al., 2007), the PFC maps represent less of the upper and lower visual field near the meridian than the IPS maps (Fig. $3 b$ ). In fact, it has been argued that the organization of the sPCS might best be described as contralateralized, not retinotopic (Jack et al., 2007). However, the substantially larger response fields in PFC neurons (Mohler et al., 1973), compared with IPS neurons (Blatt et al., 1990), may influence the distribution of voxels representing the visual field near the meridian. Pooling would be exacerbated in topographic areas with large receptive fields, like the PFC. Overall, our methods allowed us to identify four topographic areas in PPC and two in PFC that we will now consider to be candidate priority maps, because they possess a hallmark feature of theoretical priority maps: a spatial topographic organization.

\section{Event-related studies of spatial cognition}

Psychophysics and oculomotor behavior

The experimental tasks were made challenging with the use of adaptive staircases that maintained performance $\sim 70 \%$ accuracy (working memory, range of $68-72 \%$; attention, range of $65-$ $74 \%)$. The use of a staircase to control behavior was not possible in the motor intention task, and subjects had no difficulty generating accurate saccades to the visual targets after the preparation delay. On $9 \%$ of trials (range of $0-14 \%$ ), subjects made a brief uninstructed saccade during the delay period, followed by rapid refixation. Similarly, subjects made brief, most often very small, uninstructed and immediately corrected eye movements during the delay period on $8 \%$ of working memory trials (range of $0-14 \%$ ) and $7 \%$ of attention trials (range of 3-13\%). These behavioral data indicate that the four experienced subjects performed as instructed on the three spatial cognitive tasks.
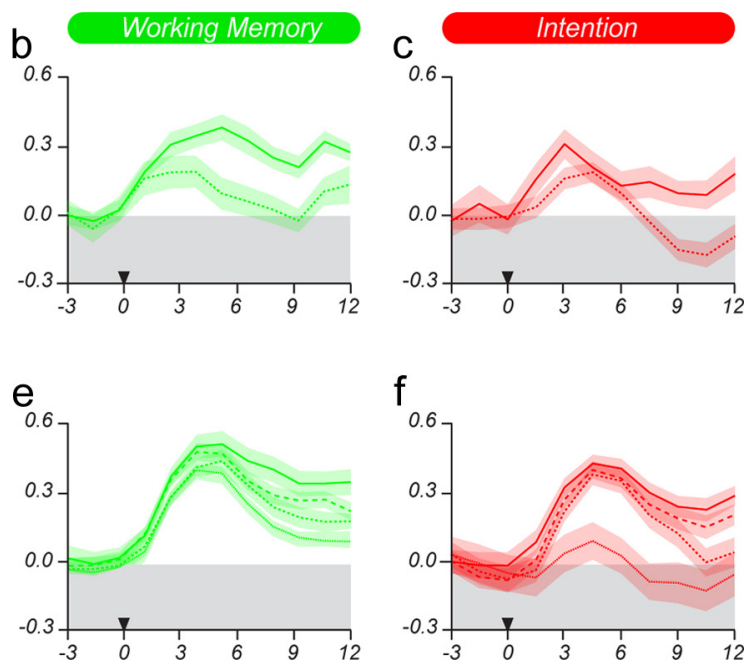

Figure 4. Subject-averaged BOLD time courses time-locked to the cue (black triangle) showing persistent delay period activity. Persistent activity in candidate priority maps

Next, we compared the delay period time courses from the candidate priority maps while subjects maintained a spatial location in working memory, attention covertly on a peripheral target, or an eye movement plan. All else being equal (i.e., the spatial priority), a theoretical priority map should not distinguish between these delay periods. In Figure 4, we plot the time courses of BOLD responses averaged across subjects from IPS0-IPS3 and sPCS/ iPCS for trials in which the contralateral visual field was prioritized for each of the three spatial cognitive tasks. In PFC, topographic sPCS showed BOLD activity that, after a large initial transient response time locked to the sample/cue, persisted throughout the entire delay period well above pretrial baseline during all three spatial cognitive tasks (Fig. $4 a-c$ ). The evidence for persistent activity in iPCS was less compelling, and, statistically, the delay period activity was greater in sPCS than iPCS across the three tasks $\left(F_{(1,3)}=21.93, p<0.02\right)$. Moreover, the temporal dynamics appear indistinguishable across the three tasks in the sPCS but less so in iPCS. In PPC, the topographic IPS areas showed two main effects. First, BOLD activity in IPS2 and IPS3 clearly persisted above baseline during all three tasks, and the temporal dynamics appear indistinguishable (Fig. $4 d-f$ ). In IPS0, and to a lesser degree IPS1, the BOLD responses during the attention and intention delays returned to pretrial baseline levels after the initial transient response. Second, the magnitude of delay period activity increased in the posterior-to-anterior direction along the IPS (i.e., IPS $0<$ IPS $1<$ IPS $2<$ IPS3) in all three tasks. A repeated-measures ANOVA confirmed a significant linear increase in delay period activity from IPS0 to IPS3 across the three tasks $\left(F_{(3,1)}=11.72, p<0.04\right)$.

Contralateral bias of persistent activity

We next tested the hypothesis that the activity in candidate topographic maps should be biased toward prioritized contralateralized space. To quantify laterality biases, we computed CIs for each subject in each topographic area for each spatial cognitive task using only the BOLD data from the delay periods (Fig. 5). In the covert attention task, all of the topographic regions showed a CI significantly greater than zero ( $t$ values ranged from 2.4 in IPS 3 to 6.2 in IPS2; all $p$ values $<0.05$ ), except for iPCS, which was 


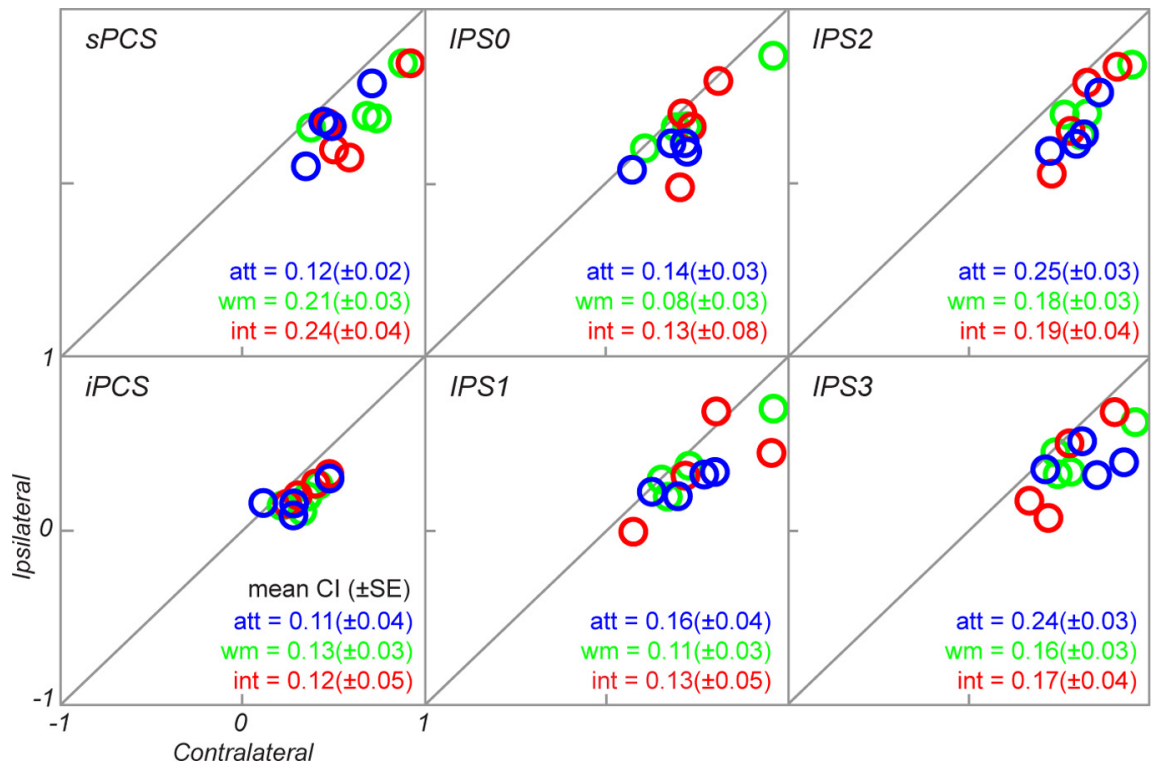

Figure 5. Contralaterality bias. For each of the tasks, the mean level of BOLD activity during the delay period for trials in which priority is ipsilateral is plotted against the mean activity during trials in which priority is contralateral to the hemisphere of the topographic area. Each circle represents a subject: blue, attention trials (att); green, working memory trials (wm); red, motor intention trials (int). Circles below the diagonal line indicate a contralateral bias. Across-subject mean Cls are given in each cell for each topographic area.

marginally significant $\left(t_{(3)}=2.1, p=0.07\right)$. In the working memory task, all CIs were significantly greater than zero ( $t$ values ranged from 2.7 in IPS 1 to 4.2 in iPCS; all $p$ values $<0.05$ ), except for IPS0 $\left(t_{(3)}=1.4, p=0.12\right)$. In the motor intention task, all CIs were significantly greater than zero ( $t$ values ranged from 2.5 in IPS3 to 3.7 in sPCS; all $p$ values $<0.05)$, except for IPS0 $\left(t_{(3)}=1.4\right.$, $p=0.13)$ and IPS1 $\left(t_{(3)}=1.2, p=0.16\right)$. Overall, the contralateral, compared with ipsilateral, hemisphere showed greater delay period activity, which was most consistently seen in sPCS, IPS2, and IPS3.

\section{Decoding spatial priority}

We used logistic regression to train classifiers to discriminate multivoxel patterns of activity associated with prioritized left versus right visual field within topographically defined candidate priority maps. Priority was defined as the hemifield that contained the memorandum in the working memory task, the locus of covert attention in the attention task, and the goal of the planned saccade in the motor intention task. The performance of the classifiers was tested by comparing their ability to decode the prioritized visual field with independent data not used in the training with null distributions of performance generated by randomly shuffling the prioritized locations and rerunning the decoding (Fig. 6, gray bars). The pattern of delay period activity in many of the topographic areas could be used to successfully decode the prioritized visual field within task (e.g., a classifier trained to discriminate the locus of covert attention was tested on its ability to predict the locus of covert attention) (Fig. 6). The multivoxel patterns of delay period activity in areas sPCS, IPS2, and IPS3 were the best predictors of the prioritized visual field for each of the three spatial cognitive tasks. The prediction accuracies in the other topographic regions were mostly at chance, with an occasional subject showing a significant level of classification. We consider the within-task decoding results simply a proof for the feasibility of our methods. Although these results do narrow our search to those three candidates, they do not directly test our main hypothesis.
Critically, we wanted to know whether the activities in priority maps are interchangeable across various spatial tasks. More generally, are they agnostic with regard to the nature of the priority? Priority map theory predicts that they only tag the spatial coordinates of prioritized locations. To directly test this, we conducted across-task decoding analyses. We trained classifiers on one task and tested their ability to generalize to the other two tasks (e.g., a classifier trained to discriminate the locus of covert attention was tested on its ability to predict a location maintained in working memory). Both sPCS and IPS2, but not other candidate areas, were able to predict priority across the three tasks (Fig. 6). Remarkably, in these areas, the percentage of correctly decoded trials only dropped slightly-a couple of percentage points - from the percentage obtained during the within-task decoding. Across all three tasks, the classifier was able to generalize and predict the prioritized visual field based on the pattern of multivoxel delay period activity regardless of the task on which it was trained.

Given that the magnitude of delay period activity was greater in the contralateral hemisphere than the ipsilateral hemisphere, it is possible that the classifiers were simply using this bias to estimate the prioritized hemifield. We think that this is not the case. First, all of the topographic areas showed a contralateral bias, but the pattern of delay period activity in only two areas was predictive of priority, regardless of cognitive task. Second, we conducted control analyses to test whether an overall contralateral bias could account for the classifier results. In each topographic area, we averaged the BOLD signal during the delay across all voxels in each hemisphere. We used the difference between the right and left hemispheres to predict which hemifield was prioritized, taking the larger of the two to predict the opposite hemifield. Although most of the predictions were numerically greater than chance, they did not reach significance and they did not come close to matching the accuracy of predictions made using the spatial pattern of multivoxel delay period activity (Fig. 6, black horizontal lines). Therefore, these results suggest that the spatial pattern of delay period activity across the topographic sPCS and IPS2 is interchangeable across the three spatial cognitive tasks.

\section{Discussion}

Priority maps are theorized to be composed of populations of neurons organized topographically (e.g., retinal) into a twodimensional map of gaze-centered space (Itti and Koch, 2001). In human cortex, fMRI studies have identified $>12$ topographic maps in visual cortex that provide an orderly tiling of visual space (Wandell et al., 2007). Recently, topographic maps have been found outside of the occipital lobes. Several topographic maps have been described in parietal cortex (Silver et al., 2005; Kastner et al., 2007; Swisher et al., 2007) and in frontal cortex (Hagler and Sereno, 2006; Kastner et al., 2007), making them ideal candidates. Using phase-encoded fMRI methods, we identified four reliable topographic maps, IPS0-IPS3, along the IPS that match those identified in previous studies, including IPS0/V7 (Tootell et al., 
1998), IPS1 and IPS2 (Sereno et al., 2001; Schluppeck et al., 2005; Silver et al., 2005), and IPS3 (Swisher et al., 2007; Konen and Kastner, 2008). Beginning in V1 and terminating at the rostral end of the IPS, these topographic maps form a strip of consecutive alternating inverted and upright representations of the contralateral visual field. In PFC, we identified two reliable topographic maps in sPCS/iPCS that are consistent with previous reports (Hagler and Sereno, 2006; Kastner et al., 2007). Given the spatial topography, we consider these as candidate priority maps.

Theoretically, prioritized maps of space may only contain information about the locations of salient and behaviorally relevant information. A location may be prioritized for a variety of reasons; for example, it may be the location of a maintained working memory representation, the location of an attended object, or the goal of an intended eye movement. Priority maps may not represent these reasons. For instance, consider a working memory delay in which one maintains the position of a stimulus that is $10^{\circ}$ to the left. In terms of an attractor dynamics model, an attractor positioned within a topographic map at the cued location could be used to represent the prioritized spatial location (Compte et al., 2000). This map may be identical to a map during the maintenance of covert attention that is $10^{\circ}$ to the left and to a map during the planning of a saccade that is $10^{\circ}$ to the left. The pattern of activity within the map of space may thus be agnostic with regard to the conditions that led to the priority of the map. Other brain areas could then read out the general map of prioritized space to fulfill the specific behavioral demands. For instance, downstream oculomotor areas (e.g., superior colliculus and brainstem saccade generator) may read out the priority maps of space in the PFC and PPC to convert eye-centered retinotopic representations into the motor metrics for both visual- and memory-guided saccades (Sommer and Wurtz, 2001; Bisley and Goldberg, 2003b). In addition, a readout of the same priority map by posterior visual areas may bias the competition for neural representation toward neurons whose receptive fields match the peaks in the priority maps (Moore and Armstrong, 2003; Gregoriou et al., 2009).

We predicted that, if priority maps only represent the location, not the cause, of priority, spatiotemporal patterns of neural activity in priority maps would be indistinguishable across the different spatial cognitive tasks. Here, we found that activity in topographic IPS2 and sPCS persisted during the maintenance of a spatial location during
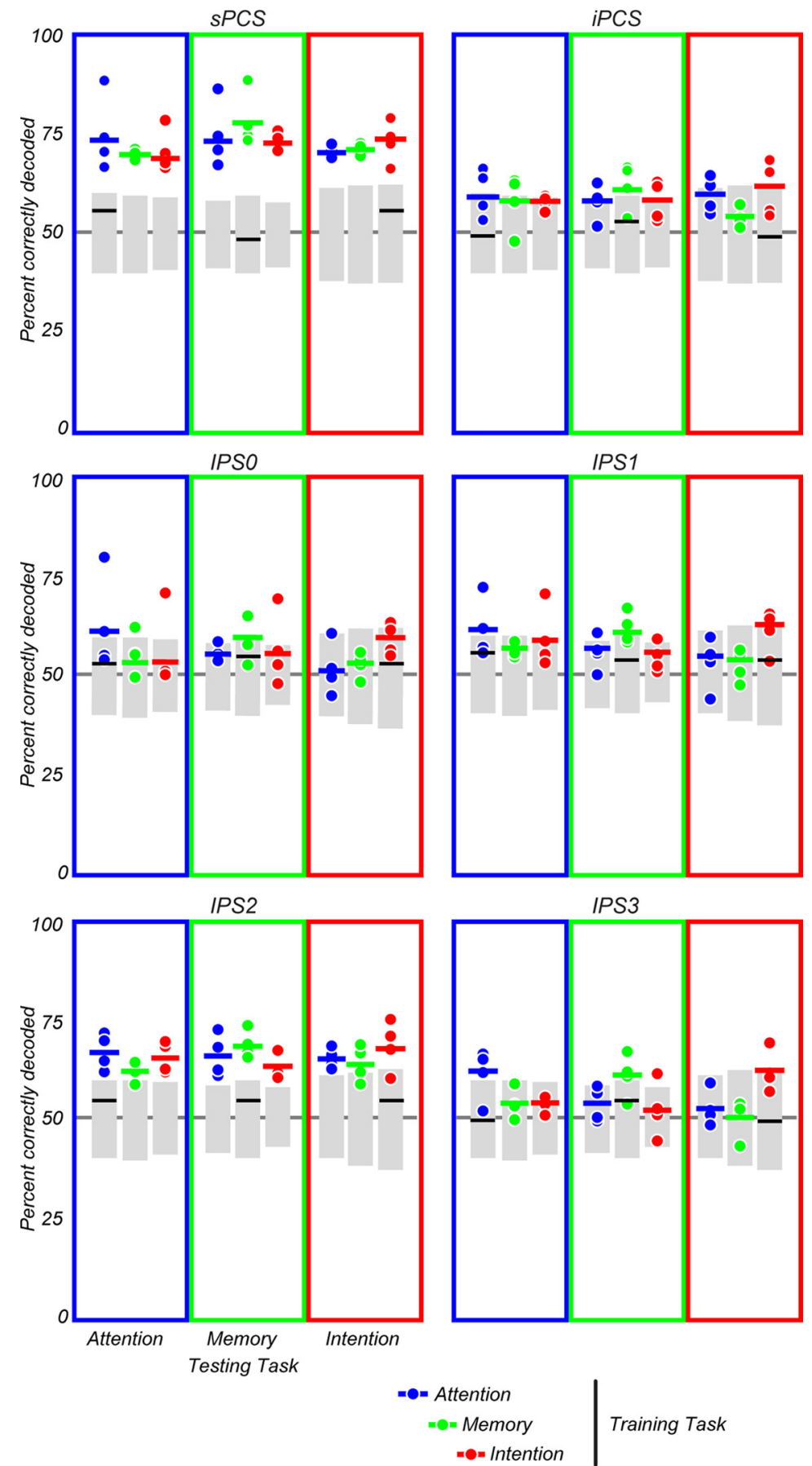

Figure 6. Classifier results for decoding the prioritized visual field. For each topographic area, the percentage of correctly decoded trials is plotted. Each dot is an individual subject, and each horizontal line is the mean performance across subjects. The color of the dots keys the task used to train the classifier. The color of the boxes keys the task used to test the classifier. Within-task classification, Dot and box colors match; across-task classification, dot and box color do not match. The gray boxes represent the 2.5th and 97.5 th percentile of the null distribution generated by random permutation analysis. Dots and bars beyond these cutoffs are significantly different from chance. The multivoxel pattern of delay period activity only in sPCS and IPS2 predicts the prioritized hemifield both within and across the three spatial cognitive tasks. The black horizontal bars are the mean performance of the control analyses, in which the mean signal difference of all voxels in the left and right hemisphere topographic areas was used to predict the prioritized hemifield. working memory, covert attention, and motor planning. These results can be interpreted in the context of the priority map theory. Priority sculpts the population activity of neurons in the topographic maps. The activities of neurons whose response fields match the prioritized location persist through- 
out the delay period as a means to maintain the prioritized location, regardless of the task demands that led to the priority.

Although the temporal dynamics of the delay period activity in sPCS and IPS2 were virtually identical across the various spatial cognitive tasks, are they interchangeable during the maintenance of a location relevant for working memory, attention, and motor intention? To answer this question, we used pattern classification to test whether spatial priority could be decoded from the multivoxel delay period activity within topographically defined candidate priority maps (Kriegeskorte, 2011). Remarkably, decoders trained to predict the locations on one task (e.g., working memory) cross-predicted the locations on other tasks (e.g., attention), suggesting that these patterns of maintenance activity in IPS2 and sPCS may be interchangeable across the tasks. Such properties make these two areas in human frontal and parietal cortex viable priority map candidates. These results strongly support the prediction from priority map theory that the location, not cause, of priority is represented in the pattern of topographic activity.

The dorsal IPS in the PPC and sPCS in the PFC consistently show the most robust delay period activity across subjects, tasks, studies, and laboratories in human neuroimaging studies of spatial working memory, spatial attention, and saccade control (Ikkai and Curtis, 2011). These two areas contain the likely human homologs or evolved variants of monkey areas lateral intraparietal area (LIP) and frontal eye field (FEF). The topographic sPCS area may correspond to the monkey FEF. Electrical stimulation of this area in humans induces saccades to the contralateral visual field (Blanke et al., 1999) and lesions disrupt contraversive saccades (Rivaud et al., 1994; Gaymard et al., 1999). Moreover, robust and spatially selective BOLD activity in human sPCS is correlated with the selection, preparation, maintenance, and generation of saccades (Corbetta et al., 1998; Curtis et al., 2004; Connolly et al., 2005; Ford et al., 2005; Curtis and Connolly, 2008). Neurons in monkey FEF have direction-selective presaccadic activity, and saccades can be elicited with little current (Bruce et al., 1985). FEF is thought to convert visual signals into potential saccade goals (Schall and Hanes, 1993). These same mechanisms may guide attention in the absence of eye movements (Thompson et al., 2005; Awh et al., 2006) and may be the means by which spatial representations are maintained in working memory (Armstrong et al., 2009). A clear topographic gradient of saccade amplitudes exists along the long axis of the FEF, with largeramplitude saccades being more numerous in dorsal FEF and smaller-amplitude saccades being more numerous in ventral FEF (Bruce et al., 1985). Additionally, FEF neurons represent all saccade directions, but it is unknown whether they are topographically organized by angle. Bruce et al. (1985) reported that saccades of similar angles were elicited by microstimulation of nearby neurons, and angle seemed to systematically progress from the lip to the fundus of the arcuate sulcus. Although here we report an angular topographic map in putative human FEF, functional imaging studies have yet to find a topographic map of amplitude in this area.

The topographic IPS2 area likely corresponds to the human homolog of the monkey LIP. In the monkey, electrophysiological (Ben Hamed et al., 2001) and fMRI (Arcaro et al., 2011) data have provided evidence for a topographic map of contralateral visual space in area LIP. Given that monkey area LIP is situated in mid-IPS and contains an inverted visual field representation, it may correspond to IPS2 in the human, which is also located in mid-IPS and contains an inverted visual field representation.
Neurons in area LIP, like FEF, increase their rate of firing when saccades are planned into their receptive field (Barash et al., 1991; Ipata et al., 2006). Moreover, their activity persists throughout spatial working memory retention intervals (Gnadt and Andersen, 1988) and during the maintenance of covert attention (Bisley and Goldberg, 2003a). Functional imaging studies of spatial cognition often activate a large portion of the IPS that likely includes IPS2 (Astafiev et al., 2003; Schluppeck et al., 2006; Serences and Yantis, 2007; Ikkai and Curtis, 2008; Srimal and Curtis, 2008). Both FEF and LIP are densely interconnected with visual and oculomotor structures (Cavada and Goldman-Rakic, 1989a, 1989b; Petrides and Pandya, 2006). Thus, they are ideally situated to receive the kind of inputs necessary to construct a priority map that could be accessed by many brain areas to influence a variety of spatially guided behaviors.

\section{References}

Arcaro MJ, Pinsk MA, Li X, Kastner S (2011) Visuotopic organization of macaque posterior parietal cortex: a functional magnetic resonance imaging study. J Neurosci 31:2064-2078. CrossRef Medline

Armstrong KM, Chang MH, Moore T (2009) Selection and maintenance of spatial information by frontal eye field neurons. J Neurosci 29:1562115629. CrossRef Medline

Astafiev SV, Shulman GL, Stanley CM, Snyder AZ, Van Essen DC, Corbetta M (2003) Functional organization of human intraparietal and frontal cortex for attending, looking, and pointing. J Neurosci 23:4689-4699. Medline

Awh E, Armstrong KM, Moore T (2006) Visual and oculomotor selection: links, causes and implications for spatial attention. Trends Cogn Sci 10: 124-130. CrossRef Medline

Barash S, Bracewell RM, Fogassi L, Gnadt JW, Andersen RA (1991) Saccaderelated activity in the lateral intraparietal area. II. Spatial properties. J Neurophysiol 66:1109-1124. Medline

Ben Hamed S, Duhamel JR, Bremmer F, Graf W (2001) Representation of the visual field in the lateral intraparietal area of macaque monkeys: a quantitative receptive field analysis. Exp Brain Res 140:127-144. CrossRef Medline

Bisley JW, Goldberg ME (2003a) Neuronal activity in the lateral intraparietal area and spatial attention. Science 299:81-86. CrossRef Medline

Bisley JW, Goldberg ME (2003b) The role of the parietal cortex in the neural processing of saccadic eye movements. Adv Neurol 93:141-157. Medline

Bisley JW, Goldberg ME (2010) Attention, intention, and priority in the parietal lobe. Annu Rev Neurosci 33:1-21. CrossRef Medline

Blanke O, Morand S, Thut G, Michel CM, Spinelli L, Landis T, Seeck M (1999) Visual activity in the human frontal eye field. Neuroreport 10: 925-930. CrossRef Medline

Blatt GJ, Andersen RA, Stoner GR (1990) Visual receptive field organization and cortico-cortical connections of the lateral intraparietal area (area LIP) in the macaque. J Comp Neurol 299:421-445. CrossRef Medline

Bruce CJ, Goldberg ME, Bushnell MC, Stanton GB (1985) Primate frontal eye fields. II. Physiological and anatomical correlates of electrically evoked eye movements. J Neurophysiol 54:714-734. Medline

Cavada C, Goldman-Rakic PS (1989a) Posterior parietal cortex in rhesus monkey. I. Parcellation of areas based on distinctive limbic and sensory corticocortical connections. J Comp Neurol 287:393-421. CrossRef Medline

Cavada C, Goldman-Rakic PS (1989b) Posterior parietal cortex in rhesus monkey. II. Evidence for segregated corticocortical networks linking sensory and limbic areas with the frontal lobe. J Comp Neurol 287:422-445. CrossRef Medline

Compte A, Brunel N, Goldman-Rakic PS, Wang XJ (2000) Synaptic mechanisms and network dynamics underlying spatial working memory in a cortical network model. Cereb Cortex 10:910-923. CrossRef Medline

Connolly JD, Goodale MA, Goltz HC, Munoz DP (2005) fMRI activation in the human frontal eye field is correlated with saccadic reaction time. J Neurophysiol 94:605-611. CrossRef Medline

Corbetta M, Akbudak E, Conturo TE, Snyder AZ, Ollinger JM, Drury HA, Linenweber MR, Petersen SE, Raichle ME, Van Essen DC, Shulman GL (1998) A common network of functional areas for attention and eye movements. Neuron 21:761-773. CrossRef Medline 
Curtis CE, Connolly JD (2008) Saccade preparation signals in the human frontal and parietal cortices. J Neurophysiol 99:133-145. CrossRef Medline

Curtis CE, Rao VY, D'Esposito M (2004) Maintenance of spatial and motor codes during oculomotor delayed response tasks. J Neurosci 24:3944-3952. CrossRef Medline

Engel SA, Rumelhart DE, Wandell BA, Lee AT, Glover GH, Chichilnisky EJ, Shadlen MN (1994) fMRI of human visual cortex. Nature 369:525. CrossRef Medline

Engel SA, Glover GH, Wandell BA (1997) Retinotopic organization in human visual cortex and the spatial precision of functional MRI. Cereb Cortex 7:181-192. CrossRef Medline

Fecteau JH, Munoz DP (2006) Salience, relevance, and firing: a priority map for target selection. Trends Cogn Sci 10:382-390. CrossRef Medline

Ford KA, Goltz HC, Brown MR, Everling S (2005) Neural processes associated with antisaccade task performance investigated with event-related FMRI. J Neurophysiol 94:429-440. CrossRef Medline

Gaymard B, Ploner CJ, Rivaud-Péchoux S, Pierrot-Deseilligny C (1999) The frontal eye field is involved in spatial short-term memory but not in reflexive saccade inhibition. Exp Brain Res 129:288-301. CrossRef Medline

Gnadt JW, Andersen RA (1988) Memory related motor planning activity in posterior parietal cortex of macaque. Exp Brain Res 70:216-220. Medline

Gregoriou GG, Gotts SJ, Zhou H, Desimone R (2009) High-frequency, long-range coupling between prefrontal and visual cortex during attention. Science 324:1207-1210. CrossRef Medline

Hagler DJ Jr, Sereno MI (2006) Spatial maps in frontal and prefrontal cortex. Neuroimage 29:567-577. CrossRef Medline

Ikkai A, Curtis CE (2008) Cortical activity time locked to the shift and maintenance of spatial attention. Cereb Cortex 18:1384-1394. CrossRef Medline

Ikkai A, Curtis CE (2011) Common neural mechanisms supporting spatial working memory, attention and motor intention. Neuropsychologia 49: 1428-1434. CrossRef Medline

Ipata AE, Gee AL, Goldberg ME, Bisley JW (2006) Activity in the lateral intraparietal area predicts the goal and latency of saccades in a freeviewing visual search task. J Neurosci 26:3656-3661. CrossRef Medline

Itti L, Koch C (2001) Computational modelling of visual attention. Nat Rev Neurosci 2:194-203. CrossRef Medline

Jack AI, Patel GH, Astafiev SV, Snyder AZ, Akbudak E, Shulman GL, Corbetta M (2007) Changing human visual field organization from early visual to extra-occipital cortex. PLoS One 2:e452. CrossRef Medline

Jammalamadaka RS, Sengupta A (2001) Topics in circular statistics. Singapore: World Scientific Publishing.

Kastner S, DeSimone K, Konen CS, Szczepanski SM, Weiner KS, Schneider KA (2007) Topographic maps in human frontal cortex revealed in memory-guided saccade and spatial working-memory tasks. J Neurophysiol 97:3494-3507. CrossRef Medline

Konen CS, Kastner S (2008) Representation of eye movements and stimulus motion in topographically organized areas of human posterior parietal cortex. J Neurosci 28:8361-8375. CrossRef Medline

Kriegeskorte N (2011) Pattern-information analysis: from stimulus decoding to computational-model testing. Neuroimage 56:411-421. CrossRef Medline

Larsson J, Heeger DJ (2006) Two retinotopic visual areas in human lateral occipital cortex. J Neurosci 26:13128-13142. CrossRef Medline

Mohler CW, Goldberg ME, Wurtz RH (1973) Visual receptive fields of frontal eye field neurons. Brain Res 61:385-389. CrossRef Medline

Moore T, Armstrong KM (2003) Selective gating of visual signals by microstimulation of frontal cortex. Nature 421:370-373. CrossRef Medline

Oldfield RC (1971) The assessment and analysis of handedness: the Edinburgh inventory. Neuropsychologia 9:97-113. CrossRef Medline
Pereira F, Mitchell T, Botvinick M (2009) Machine learning classifiers and fMRI: a tutorial overview. Neuroimage 45:S199-S209. CrossRef Medline

Petrides M, Pandya DN (2006) Efferent association pathways originating in the caudal prefrontal cortex in the macaque monkey. J Comp Neurol 498:227-251. CrossRef Medline

Press WA, Brewer AA, Dougherty RF, Wade AR, Wandell BA (2001) Visual areas and spatial summation in human visual cortex. Vision Res 41:13211332. CrossRef Medline

Rivaud S, Müri RM, Gaymard B, Vermersch AI, Pierrot-Deseilligny C (1994) Eye movement disorders after frontal eye field lesions in humans. Exp Brain Res 102:110-120. CrossRef Medline

Schall JD, Hanes DP (1993) Neural basis of saccade target selection in frontal eye field during visual search. Nature 366:467-469. CrossRef Medline

Schluppeck D, Glimcher P, Heeger DJ (2005) Topographic organization for delayed saccades in human posterior parietal cortex. J Neurophysiol 94: 1372-1384. CrossRef Medline

Schluppeck D, Curtis CE, Glimcher PW, Heeger DJ (2006) Sustained activity in topographic areas of human posterior parietal cortex during memory-guided saccades. J Neurosci 26:5098-5108. CrossRef Medline

Schneider KA, Richter MC, Kastner S (2004) Retinotopic organization and functional subdivisions of the human lateral geniculate nucleus: a highresolution functional magnetic resonance imaging study. J Neurosci 24: 8975-8985. CrossRef Medline

Serences JT, Yantis S (2006) Selective visual attention and perceptual coherence. Trends Cogn Sci 10:38-45. CrossRef Medline

Serences JT, Yantis S (2007) Spatially selective representations of voluntary and stimulus-driven attentional priority in human occipital, parietal, and frontal cortex. Cereb Cortex 17:284-293. CrossRef Medline

Sereno MI, Dale AM, Reppas JB, Kwong KK, Belliveau JW, Brady TJ, Rosen BR, Tootell RB (1995) Borders of multiple visual areas in humans revealed by functional magnetic resonance imaging. Science 268:889-893. CrossRef Medline

Sereno MI, Pitzalis S, Martinez A (2001) Mapping of contralateral space in retinotopic coordinates by a parietal cortical area in humans. Science 294:1350-1354. CrossRef Medline

Silver MA, Ress D, Heeger DJ (2005) Topographic maps of visual spatial attention in human parietal cortex. J Neurophysiol 94:1358-1371. CrossRef Medline

Sommer MA, Wurtz RH (2001) Frontal eye field sends delay activity related to movement, memory, and vision to the superior colliculus. J Neurophysiol 85:1673-1685. Medline

Srimal R, Curtis CE (2008) Persistent neural activity during the maintenance of spatial position in working memory. Neuroimage 39:455-468. CrossRef Medline

Swisher JD, Halko MA, Merabet LB, McMains SA, Somers DC (2007) Visual topography of human intraparietal sulcus. J Neurosci 27:5326-5337. CrossRef Medline

Thompson KG, Bichot NP (2005) A visual salience map in the primate frontal eye field. Prog Brain Res 147:251-262. CrossRef Medline

Thompson KG, Biscoe KL, Sato TR (2005) Neuronal basis of covert spatial attention in the frontal eye field. J Neurosci 25:9479-9487. CrossRef Medline

Tootell RB, Hadjikhani N, Hall EK, Marrett S, Vanduffel W, Vaughan JT, Dale AM (1998) The retinotopy of visual spatial attention. Neuron 21: 1409-1422. CrossRef Medline

Wandell BA, Dumoulin SO, Brewer AA (2007) Visual field maps in human cortex. Neuron 56:366-383. CrossRef Medline

Wetherill GB, Levitt H (1965) Sequential estimation of points on a psychometric function. Br J Math Stat Psychol 18:1-10. CrossRef Medline

Winawer J, Horiguchi H, Sayres RA, Amano K, Wandell BA (2010) Mapping hV4 and ventral occipital cortex: the venous eclipse. J Vis 10:1. CrossRef Medline 